\title{
The Changing Therapeutic Landscape in Metastatic Prostate Cancer
}

\author{
Alan J Koletsky \\ Lynn Cancer Institute, Boca Raton, FL, US
}

DOl: https://doi.org/10.17925/OHR.2017.13.02.112

O

ver the past several years a number of novel and diverse agents have provided a significant clinical benefit for patients with metastatic castration-resistant prostate cancer including abiraterone, enzalutamide, sipuleucel-T, cabazitaxel, and radium-223. The early use of docetaxel or abiraterone at initiation of standard androgen deprivation therapy in patients with metastatic hormone-sensitive prostate cancer has also led to substantial improvements in overall survival. The identification of a truncating mutation in the androgen receptor (ARV7), a biomarker of resistance, may help clarify a more optimal sequencing of hormonal and chemotherapy-based therapies for patients with metastatic disease. The genomic landscape of both primary and metastatic prostate cancer has been an important focal point of translational research. The most widely studied pathways that affect tumorigenesis are the phosphoinositide 3-kinase (PI3K)/phosphatase and tensin homolog (PTEN)/protein kinase B (AKT) and poly ADP ribose polymerase (PARP) and DNA repair pathways. This review will highlight recent clinical trials which have had a major impact on the management of patients with metastatic disease with an emphasis on treatments driven by common genomic aberrations present in advanced prostate cancer.

\section{Keywords}

Metastatic hormone-sensitive prostate cancer (mHSPC), metastatic castrationresistant prostate cancer (MCRPC), androgen receptor (AR), androgen deprivation therapy (ADT), radiographic progression-free survival (rPFS), failure-free survival (FFS), prostatespecific antigen (PSA), prostrate specific membrane antigen (PSMA), granulocytemacrophage colony stimulating factor (GM-CSF), radiographic skeletal-related events (rSRE)

Disclosure: Alan J Koletsky serves on speaker's bureaus for Bristol Myers Squibb, Exelixis, and Astellas. No funding was received for the publication of this article.

Compliance with Ethics: This study involves a review of the literature and did not involve any studies with human or animal subjects performed by any of the authors.

Authorship: All named authors meet the International Committee of Medical Journal Editors (ICMJE) criteria for authorship of this manuscript, take responsibility for the integrity of the work as a whole, and have given final approval to the version to be published.

Open Access: This article is published under the Creative Commons Attribution Noncommercial License, which permits any noncommercial use, distribution, adaptation, and reproduction provided the original author(s) and source are given appropriate credit.

Received: August 21, 2017

Accepted: September 25, 2017

Citation: Oncology \& Hematology Review, 2017;13(2):112-6

Corresponding Author: Alan J Koletsky, MD, Lynn Cancer Institute, 701 NW 13th Street, Boca Raton, FL 33486, US.
Androgen ablation therapy (ADT) alone has long been the standard of care (SOC) for patients with metastatic prostate cancer initiating systemic therapy. Although most patients treated with standard ADT achieve a substantial prostate specific antigen (PSA) decline, transition to a castration-resistant state invariably occurs. In a large retrospective review of 659 patients with primary advanced prostate cancer and bone metastasis, the median duration of response to initial ADT was 22.4 months. ${ }^{1}$ Risk factors associated with a shorter overall survival (OS) in men treated with ADT alone included pretreatment PSA level, Gleason score, and extent of bone involvement at diagnosis.

\section{Chemo-hormonal therapy in metastatic hormone-sensitive prostate cancer-the CHAARTED and STAMPEDE trials}

Early studies which sought to examine a potential efficacy of concurrent ADT and chemotherapy were limited by the small numbers of patients enrolled, many of whom had a low tumor burden at study entry. ${ }^{2,3}$ In the CHAARTED trial, 790 metastatic hormone-sensitive patients (mHSPC) were randomized to receive ADT alone or ADT plus docetaxel. Patients were prospectively stratified into high volume disease (defined as four or more bone metastases with at least one outside the vertebral column and pelvis and/or visceral metastases) and low-volume disease. ${ }^{4}$ Long-term results from the CHAARTED trial were presented at ESMO 2016. Patients receiving ADT plus docetaxel had a significant improvement in median OS compared with ADT alone (57.6 months versus 42 months; hazard ratio [HR] 0.73; $p=0.0018$ ). A subgroup analysis continued to show benefit from the addition of docetaxel only in the high volume group. The median OS in this group was 51.2 months for ADT plus docetaxel versus 34.4 months for ADT alone (HR 0.61; $p<0.0001$ ). Secondary end-points including time to clinical progression and quality of life (Functional Assessment of Cancer Therapy-Prostate [FACT-P] questionnaire) also benefited the group with high-volume disease. An OS benefit with earlier use of docetaxel and ADT in patients with low volume disease has yet to be demonstrated with longer follow-up; although, these patients had a longer time to onset of CRPC, PSA progression, and clinical progression.

The STAMPEDE trial included both non-metastatic (MO) and metastatic hormone-naive (M1) patients in two of the comparator arms each randomized to ADT plus chemotherapy (with or without zoledronic acid) and compared to a control group receiving ADT alone., ${ }^{5,6} \mathrm{~A}$ total of 592 men with newly diagnosed M1 disease were accrued to the ADT plus docetaxel arm of STAMPEDE. Docetaxel was started within 2 weeks of randomization, and approximately 9 weeks after initiation of ADT, similar to the time used 
in the CHAARTED trial. About $77 \%$ of patients in this cohort were able to complete all six planned cycles of docetaxel. The median OS benefit with the addition of docetaxel was approximately 10 months compared with ADT alone (81 versus 71 months). Patients who received chemotherapy had a $22 \%$ reduction in risk of all-cause death (HR 0.78). Pre-determined intermediate outcomes including prostate cancer specific survival benefit and failure-free survival (FFS) also favored the addition of docetaxel. The MO patient cohort had smaller numbers of enrolled patients with fewer deaths at last interim analysis and with an insufficient number of events to determine a potential benefit for docetaxel in this setting. Several other clinical trials have addressed the benefit of adding docetaxel to ADT for patients with hormone-sensitive high-risk non-metastatic or metastatic prostate cancer. ${ }^{7-9}$ Benefits have been seen for FFS but with contrasting results with regard to OS.

\section{Early use of abiraterone in metastatic hormone- sensitive prostate cancer-results from the LATTITUDE and STAMPEDE trials}

Abiraterone acetate (Zytiga ${ }^{\oplus ;}$; Janssen, Beerse, Belgium) blocks the production of testicular, adrenal, and intra-tumor tissue/cell androgens resulting in a decrease in downstream androgen receptor (AR) signaling. Since AR signaling is necessary for androgen sensitive cell proliferation, ${ }^{10}$ it was postulated that abiraterone would have activity in earlier stages of the disease. A phase II trial of neoadjuvant abiraterone in combination with leuprolide and prednisone prior to radical prostatectomy, showed a pathologic complete response (CR), or near $\mathrm{CR}$, in one-third of men with high-risk, localized prostate cancer.11

The results of two studies confirmed that early use of abiraterone significantly improves survival in men with metastatic hormone-sensitive prostate cancer. In the LATTITUDE phase III trial, 1,199 men with MHSPC were randomized to receive either ADT therapy plus abiraterone acetate (1,000 mg daily) plus prednisone (5 mg daily), or ADT plus dual placebo. ${ }^{12}$ Patients enrolled had at least two of the following three risk factors: Gleason score 8 or higher, three or more bone lesions, or measurable visceral metastases. The co-primary end points were OS and radiographic progression-free survival (rPFS). At the first interim analysis, after a median follow-up of 30.4 months, the median survival had not been reached in the abiraterone-treated group; the median OS in the control group was 34.7 months (HR 0.62; $\mathrm{p}<0.001$ ). The 3 year OS for patients treated with abiraterone was $66 \%$ versus $49 \%$ in the placebo arm. The median duration of rPFS was 33.0 months in the abiraterone arm versus 14.8 months in the placebo arm ( $H R$ for disease progression $=0.47 ; p<0.001$ ). Patients receiving abiraterone had higher rates of grade $3 / 4$ adverse events (AES) compared with the control arm. These included hypertension (20.3\% versus $10.0 \%$ ), hypokalemia (10.4\% versus 1.3\%), and elevated liver transaminases, alanine aminotransferase (ALT; $5.5 \%$ versus 1.3\%), and aspartate aminotransferase (AST; $4.4 \%$ versus $1.5 \%)^{12}$

In the STAMPEDE trial, 1,917 patients with newly diagnosed locally advanced or high-risk MHSPC were randomized 1:1 to receive either abiraterone acetate (1,000 mg daily) plus prednisone ( $5 \mathrm{mg}$ daily), plus standard of care (SOC) or SOC alone, defined as the administration of ADT therapy for 2 years or until disease progression..$^{13}$ At time of study entry, $95 \%$ of patients were newly diagnosed, 52\% had metastatic disease, and $88 \%$ had disease that had metastasized to bone. At a median follow-up of 40 months, 184 deaths had occurred in the abiraterone arm and 262 deaths in the SOC arm.
The median-adjusted HR for median OS was 0.63 ( $p=0.0000012)$. Patients in the abiraterone arm also had a $55 \%$ reduction in time to development of skeletal-related events (SRE). AEs occurred more frequently in patients receiving abiraterone with $41 \%$ of patients experiencing grade $3 / 4$ AES versus $29 \%$ in the SOC control arm.

Both the LATTITUDE and STAMPEDE trials showed significant risk reductions for men with newly diagnosed hormone naïve high-risk metastatic prostate cancer treated with abiraterone. The survival benefits were quite similar with a 38\% lower risk of death in the LATTITUDE and 37\% lower relative risk of death in STAMPEDE. Early treatment with abiraterone is attractive for patients with metastatic prostate cancer initiating therapy who are not fit for chemotherapy. These patients tend to be older and often have multiple co-morbidities and poor bone marrow reserve. Some may simply choose not to receive chemotherapy.

The ARASENS trial (NCT02799602) is an ongoing international phase III double blind, placebo-controlled trial in which patients with newly diagnosed metastatic HSPC are randomized 1:1 to receive ODM-201, an oral AR antagonist or placebo both with standard ADT and docetaxel. The primary endpoint is OS.

\section{Immunotherapeutic approaches for the treatment of prostate cancer}

Autologous vaccines-sipuleucel-T and the IMPACT trial Prostate cancer cells display a number of specific tumor-associated antigens, notably PSA, prostate acid phosphatase (PAP), and prostatespecific membrane antigen (PSMA), making them an attractive venue for immunotherapy. Autologous vaccines work by activating, priming, and enriching the host's immune cells for reinfusion. Sipuleucel-T was the first vaccine approved for the treatment of cancer based on the results of the confirmatory IMPACT trial. ${ }^{14}$ Patients receiving sipuleucel-T had an improvement in OS of 4.1 months compared with the placebo arm and a $22 \%$ reduction in the risk of death. The survival benefit was maintained in pre-specified subgroups that included Eastern Cooperative Oncology Group (ECOG) performance status (0 versus 1), Gleason score ( $<7$ or $>8$ ), PSA level above or below median, and number of bone metastases $(0-5,6-10,>10)$. Currently there are no surrogate biomarkers for response to this vaccine or endpoints for survival. The optimal selection of patients who benefit the most from sipuleucel-T in IMPACT has been retrospectively reviewed by Schellhammer, et al. ${ }^{15}$ The most important prognostic factor was baseline PSA. PSA levels were divided into quartiles to assess the predictive value at each quartile on OS. While all PSA quartile groups benefitted from sipuleucel-T, patients in the lowest baseline quartile (PSA <22) had the most benefit with an estimated improvement in medial survival of 13 months compared with 2.8 months in the highest quartile group (PSA >134). This supports the earlier use of sipuleucel-T in patients with metastatic disease.

\section{Viral-vector-based vaccines-PROSTVAC-VF}

PROSTVAC-VF is a poxvirus-based vaccine in which PSA is bound to co-stimulatory molecules known as TRICOM. The vaccine is injected subcutaneously and the complex is taken up by dendritic cells that activate anti-PSA T cells which target prostate cancer cells displaying membranebound PSA. The lysed cancer cells release new tumor-associated antigens which activate more $T$ cells thereby expanding the anti-tumor effect. In an early phase II study with PROSTVAC-VF, 125 patients with minimally symptomatic $\mathrm{MCRPC}$ were randomized to receive the vaccine or placebo. ${ }^{16}$ 
The primary endpoint of this study was PFS. Although this end point was not met, OS after 3 years of follow-up was increased significantly in the PROSTAC-VF treated arm by 8.5 months (25.1 versus 16.6 months: HR 0.56; $\mathrm{p}=0.0061$ ). In a subsequent phase ॥ study, PROSTVAC-VF increased PFS by more than 6 months in $63 \%$ of patients. ${ }^{17}$ There was also a significant slowing of the PSA doubling time from 5.3 months to 7.7 months. The PROSPECT Global phase III trial (NCT01322490) randomized men with non-symptomatic or minimally symptomatic MCRPC to one of three arms: PROSTVAC-VF + adjuvant granulocyte-macrophage colony stimulating factor (GM-CSF) (arm 1); PROSTVAC-VF + adjuvant placebo (arm 2), or vector placebo (adjuvant placebo) (arm 3). No cross over was allowed and patients went on to receive SOC treatment following progression. PROSPECT has completed accrual and results from this trial are expected shortly.

\section{Immune checkpoint inhibition in metastatic castration-resistant prostate cancer}

Prostate cancers have a low mutation rate and limited CD8-T cell infiltration and rarely express programmed death 1 (PD-1), unlike bladder and renal cell cancers where PD-1/programmed death-ligand 1 (PD-L1) therapy is effective. ${ }^{18} \mathrm{~A}$ phase $\mathrm{Ib}$ trial of nivolumab in 17 patients with advanced prostate cancer failed to show any objective responses. ${ }^{19}$ Bishop et al. have demonstrated that non AR-mediated upregulation of PD-L1 expression occurs in both enzalutamide-resistant pre-clinical models and patients with prostate cancer. ${ }^{20}$ In a recent phase II trial, patients who had progressed on enzalutamide were treated with the anti-PD-1 antibody pembrolizumab. ${ }^{21}$ Three of the first 10 patients treated had rapid and significant decreases in PSA from pre-treatment levels of 46,71 , and 2,503 to $<0.1$. Two of the three patients with measurable disease (one with liver metastases) had a partial response and two were able to discontinue opiate analgesics. All three patients remain free of progression at 30,55 , and 16 weeks of followup. Two of the three responders had a baseline biopsy prior to starting pembrolizumab. Biopsies from these patients showed presence of CD8 tumor infiltration and PD-L1 expression. Of the remaining seven patients, three had stable disease at 30, 47, and 50 weeks, which were ongoing, and four patients had disease progression. Expected immune-related AEs were observed. None of the responders had an immune-related $A E .^{21}$ A phase III trial in men with locally advanced or MCRPC, comparing the anti-PD-L1 antibody, atezolizumab plus enzalutamide with enzalutamide alone, is currently in progress (NCT03016312).

\section{Combination immunotherapy strategies}

Despite the lack of efficacy of anti-PD-1/PD-L1 antibody monotherapy in the treatment of $\mathrm{MCRPC}$, there is a renewed interest in exploring combination checkpoint inhibitors which can work synergistically to restore cellularmediated antitumor immunity. The cytotoxic T-lymphocyte-associated protein 4 (CTLA-4) and PD-1 pathways that inhibit both T-cell activation and T-cell function, have been the most well-defined and therapeutically active targets for checkpoint blockade..22 Ipilimumab is an immuno-stimulatory antibody that inhibits CTLA-4 activity thereby enhancing the immune system's T cell's response to cancer. In a phase III randomized clinical trial, patients with MCRPC who had progressed on docetaxel were treated with a single dose of bone-targeted radiotherapy, and then randomized to Ipilimumab at a dose of $10 \mathrm{mg} / \mathrm{kg}$ or placebo, every 3 weeks for up to four cycles.$^{23}$ Although the primary endpoint of OS was not met, patients treated with Ipilimumab had an improvement in PFS of 4 months, compared with 3.1 months in the placebo arm (HR 0.70; $p<0.001$ ), and an improved PSA response (13.1\% versus 5.2\%). Data from a pre-specified and post-hoc subgroup suggested an OS benefit for ipilimumab in the subset of patients with better prognostic factors that included absence of visceral metastases, hemoglobin $>10$, and alkaline phosphatase $<1.5$ upper limit of normal (ULN). The median OS in this subset of patients was 22.7 months with ipilimumab versus 15.8 months with placebo (HR 0.62; $\mathrm{p}=0.0038$ ).

The combination of ipilimumab and nivolimab has shown significant improvement in median PFS in patients with metastatic melanoma compared with ipilimumab monotherapy. ${ }^{24}$ In prostate cancer, Gao et al. have shown that while ipilimumab increased levels of tumor infiltrating $T$ cells, it also increased levels of other compensatory inhibitory immune checkpoint molecules including PD-L1 and VISTA in tumor specimens from untreated and treated ipilimumab patients in a pre-surgical clinical trial. ${ }^{25}$ These observations provide a rationale for combining ipilimumab and nivolumab in patients with prostate cancer, with the goal of targeting multiple inhibitory pathways. The CheckMate 650 (NCT02985957) phase II trial is evaluating preliminary safety and efficacy of this combination in men with MCRPC. A second single-arm phase II trial (NCT03061539) is assessing the efficacy of the combination in MCRPC patients who have progressed following at least one line of therapy and have a pre-specified immunogenic signature, either mismatch repair, defective DNA repair, or high inflammatory infiltrate by immunochemistry $(\mathrm{IHC})$.

Clinical trials combining vaccine therapy and other check-point inhibitors are currently in progress. Jhu et al. recently presented preliminary efficacy for the combination of sipuleucel-T and indoximod, an inhibitor of indoleamine dioxygenase (IDO), a pivotal enzyme that inhibits T-cell activation. ${ }^{26}$ The median rPFS rate in the combined arm was 10.3 months versus 4.1 months in the sipuleucel-T alone arm. A trial of PROSTVAC in combination with ipilumumab and/or nivolumab in the neo-adjuvant setting in patients with localized prostate cancer is also in progress (NCT02933255).

\section{Androgen receptor signaling and the PI3k pathway and PTEN loss in prostate cancer}

AR signaling occurs throughout the course of prostate cancer and drives cell growth and survival in early and late stages of the disease. Despite initial responses to ADT, resistance to treatment inevitably occurs due to AR reactivation and signaling, despite castrate levels of testosterone. AR gene amplification and overexpression, increased sensitivity to agonists, and ligand-independent AR reactivation mediated by AR splice variants also takes place, resulting in constitutive AR activation. Phosphoinositide 3-kinases (PI3ks) are lipid kinases that normally regulate basic cellular anabolic and catabolic processes, including protein synthesis, metabolism, proliferation, and survival. ${ }^{27}$ In prostate cancer, however, the PI3K pathway is significantly de-regulated. ${ }^{28}$ Aberrant PI3K/protein kinase B (AKT)/mechanistic target of rapamycin (mTOR) signaling is present in $70-100 \%$ of men with advanced prostate cancer, ${ }^{29}$ and loss of phosphatase and tensin homolog (PTEN) leads to activation of the phosphoketolase (PKT) pathway in approximately $60 \%$ of CRPC patients. ${ }^{30}$ PTEN pathway activation is associated with resistance to ADT, disease progression, and poor outcomes in men with prostate cancer. ${ }^{31}$ The development and clinical utility of agents that block the interaction between AR signaling and PKT pathways, with the goal of overcoming treatment resistance in men with PTEN-loss and the identification of biomarkers that can predict response to $\mathrm{PI} 3 \mathrm{~K}$ pathway inhibitors, are areas of active interest.

de Bono et al. have presented a study supporting PTEN loss as a prognostic marker for response to treatment with ipatasertib, a potent oral competitive 
inhibitor of PI3K-AKT signaling. Patients with MCRPC, previously treated with docetaxel, were randomized to ipatasertib at a dose of $400 \mathrm{mg}, 200 \mathrm{mg}$, or placebo in combination with abiraterone $1,000 \mathrm{mg}$ and prednisone 10 mg, orally, daily. ${ }^{32}$ PTEN expression was assessed by IHC and PTEN-loss by fluorescent in situ hybridization (FISH) and next generation sequencing (NGS). PTEN IHC was evaluated in 165 of the 253 patients randomized, and of these, PTEN loss was detected in 71 (41\%). Median rPFS was 7.5 months in the PTEN-loss ipatasertib plus abiraterone arm, and 5.6 months in the non-PTEN abiraterone plus placebo arm. In the PTEN-loss arm the rPFS survival benefit was greatest at the $400 \mathrm{mg}$ dose of ipatasertib. Based on these results, the combination of ipatasertib and abiraterone is further being explored in a phase III trial (IPATential150; NCT03072238).

\section{Treatment of patients with DNA repair alterations-the TOPARP-A trial}

Genomic alterations that inactivate genes involved in the repair of DNA damage through homologous recombination (PARP), are present in approximately $22.7 \%$ of men with prostate cancer. ${ }^{33}$ Men with metastatic prostate cancer and gene mutations such as PARP have sustained responses to PARP inhibitors and platinum-based chemotherapy (Figure 1).

In the pivotal TOPARP-A trial, 50 men with previously-treated MCRPC, received the oral PARP inhibitor, olaparib, at a dose of $400 \mathrm{mg}$ twice daily until radiographic progression, clinical progression, or development of serious AES. ${ }^{34}$ The primary endpoint was overall response rate (ORR) defined as either objective response, PSA decrease of $>50 \%$, or reduction in the number of circulating tumor cells. Tumor biopsies from all patients were submitted for NGS, exome transcriptome analysis, and digital polymerase chain reaction testing. Several actionable mutations that relate to DNA repair pathways were identified including BRCA1, BRCA2, ATM, CHEK2, and FANCONI anemia genes (FANCA). One or more of these mutations was present in 16 patients (33\%). BRCA2 mutations were present in seven patients (four with bi-allelic somatic loss, three with germline mutations), ATM mutations in five patients, homozygous somatic deletions of BRCA1 or CHEK2 with FANCA deletion in three patients, a somatic frameshift mutation in PALB2 (a localizer of BRCA2) in one patient with a heterozygous PALB2 deletion, and a bi-allelic somatic mutation in HDAC (which plays a role in ATM function) in one patient. Responses were seen in 14 of 16 patients (88\%) with DNA-repair gene mutations, compared with two of 33 patients $(6 \%)$ without these mutations $(p<0.001)$. Median rPFS was longer in mutation-positive patients with DNA-repair defects treated with olaparib than in the mutation-negative group ( 9.8 versus 2.7 months; $p<0.001)$ as was OS (13.8 versus 7.5 months $(p<0.05)$ The most common grade $>3$ AEs were anemia (20\%), fatigue (12\%), and leukopenia (6\%). Dose reduction of olaparib to $300 \mathrm{mg}$ twice daily was required in $26 \%$ of patients, most commonly due to anemia. AEs led to treatment discontinuation in $6 \%$ of patients. ${ }^{34}$ Based on these clinically significant results, olaparib received breakthrough designation by the US Food and Drug Administration (FDA) for the treatment of BCRA1/2 or ATM gene-mutated MCRPC in January 2016.

Several other PARP inhibitor trials involving deleterious homologous recombination DNA repair (HRR) aberrations, are planned or in progress. The PROfound trial (NCT02987543) is a randomized, phase III trial that is evaluating olaparib's efficacy and safety versus physician's choice of either abiraterone or enzalutamide. Cohort A includes patients with MCRPC and BRCA 1, BRCA2, or ATM mutations, while cohort B will include patients with
Figure 1: PARP inhibition enhances tumor response to chemotherapy

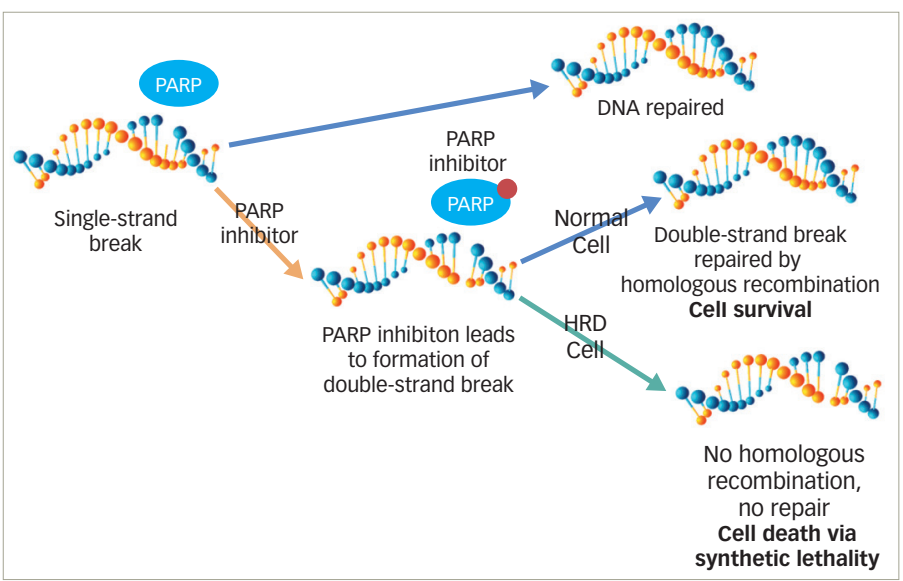

PARP facilitates repair of DNA damage from exposure to chemotherapy, radiation therapy, or ultraviolet light. Cancer cells are more dependent on PARP for DNA repair than normal cells. PARP inhibitors prevent cancer cells from effectively repairing single or double-DNA strand breaks leading to cell death. HRD = homologous recombination deficiency; $P A R P=$ poly ADP-ribose polymerase inhibitor. Reproduced with permission from Clovis Oncology.

12 other HRR genes. Patients will be randomized (2:1) to olaparib $300 \mathrm{mg}$ orally twice daily to the standard treatment arm. Treatment will be continued until radiographic progression or lack of treatment tolerability. The primary end-point is rPFS. Secondary end-points include confirmed ORR, time to pain progression, OS (all Cohort A) and rPFS (both cohorts combined). Rucaparib is an oral small molecule inhibitor of PARP that was recently approved as monotherapy for patients with BRCA2 mutation-positive advanced ovarian cancer following chemotherapy. The phase II TRITON2 trial (NCT02952534) will determine the response rate with rucaparib treatment in patients with MCRPC associated with BRCA1/2 or ATM mutations, who have progressed on both AR-targeted therapy and taxane-based chemotherapy. TRITON3 (NCT02975934) has a similar trial design to PROfound except that patients will be screened for BRCA1/2 or ATM mutations only, and subsequently randomized to receive rucaparib or abiraterone, enzalutamide or docetaxel chemotherapy, as recommended by the treating physician.

\section{Summary}

Prostate cancer is the most common cancer afflicting men in North America and is estimated to become the third leading cause of death behind lung cancer and colorectal cancers. ${ }^{35}$ Although prostate cancers are clinically heterogeneous, AR signaling occurs in all stages of the disease and suppression of the AR remains the cornerstone of therapy. The early addition of chemotherapy (docetaxel) or hormone therapy (abiraterone) to standard ADT for patients with newly diagnosed hormonesensitive metastatic disease, has resulted in a robust improvement in OS. Immunotherapeutic strategies that utilize different checkpoint inhibitors to both stimulate T-cell activation and inhibit downstream immune-modulating resistance pathways have shown clinical benefit in other solid tumor types and results from current and future clinical trials in prostate cancer are eagerly awaited to determine whether such synergy will provide additional clinical benefit.

Continued progress in transcriptional and genomic profiling has led to the identification of a number of clinically actionable mutations that are common in men with $\mathrm{MCRPC}$, thereby affording new avenues of personalized therapy 
for mutation-positive patients. In particular, genomic alterations of the PI3KAKT/mTOR signaling pathway and its interaction with AR signaling and PTEN, have led to the development of novel agents that target different kinases in this pathway and show early promise in clinical trials. Both somatic and germline aberrations in DNA repair genes can restore sensitivity to PARP inhibition, and show significant activity in men with BRCA2, BRCA1, and ATM mutations. Comparison with standard therapies used in the initial treatment of MCRPC will be needed to determine whether PI3K-AKT signaling inhibitors, and PARP inhibitors, can be utilized in the frontline setting. The analysis of genomic profiling of tumor specimens in patients with metastatic prostate cancer can be challenging at times due to lack of sufficient viable tissue particularly from sites of bone metastasis. Further refinements of liquid biopsies which analyze circulating tumor cells, and cell-free DNA may provide an attractive alternative methodology. With the anticipated identification of additional genomic aberrations that drive prostate cancer cell growth and proliferation and the potential to identify new targeted therapies or uncover new mechanisms of resistance to available agents, the therapeutic landscape in advanced prostate cancer is more diverse than ever. $\mathrm{a}$
1. Divrik RT, Turken $L$, Sahin $A F$, et al., Prediction of response to androgen deprivation therapy and castration resistance in primary metastatic prostate cancer, Urol Int, 2012;88:25-33.

2. Millikan R, Wen S, Pagliaro L et al., Phase 3 trial of androgen ablation with or without 3 cycles of systemic chemotherapy for ablation with or without 3 cycles of systemic 2008.5936-42.

3. Gravis G, Fizazi K, Joley F, et al., Androgen-deprivation therapy alone or with docetaxel in non-castrate metastatic prostate cancer (GETUG-AFU 15): a randomized, open-label, phase 3 trial, Lancet Oncol, 2013;14:149-58.

4. Sweeney CJ, Chen YH, Carducci M, et al., Chemo-hormonal therapy in Metastatic Hormone-Sensitive Prostate Cancer, N Eng J Med, 2015:373:737-46.

5. James N, M Sydes, Clarke N, et al., Addition of docetaxel, zoledronic acid, or both to first-line long term hormone therapy in prostate cancer (STAMPEDE): survival results from an adaptive multistage, platform randomized controlled trial, Lancet, 2016;387:1163-77.

6. Carthon B, Antonarakis E, The STAMPEDE trial: paradigm-changing data through innovative trial design, Trans Cancer Res, 2016:1-10.

Gravis G, Boher J, Joley F, et al., Androgen deprivation therapy (ADT) plus docetaxel versus ADT alone in metastatic non castrate prostate cancer: impact of metastatic burden and long term survival analysis of the randomized phase 3 GETUG-AFU 15 trial, Eur Urol, 2016;70:256-62.

8. Fizazi K, Faivr L, Lesaunier F, et al., Androgen deprivation therapy plus docetaxel and estramustine versus androgen deprivation therapy alone for high risk localized prostate cancer (GETUG 12), a phase 3 randomized controlled trial, Lancet Oncol, 2015;16:787-94.

9. Schweizer M, Huang P, Kattan M, et al., Adjuvant leuprolide with or without docetaxel in patients with high-risk prostate cance after radical prostatectomy (TAX 3501): important lessons for future trials, Cancer, 2013;119:1360.

10. Yanf Q, Fung K, Day W, et al., Androgen receptor signaling is required for androgen-sensitive human prostate cancer cel proliferation and survival, Cancer Cell Int, 2005:5:5-8.

11. Taplin M, Montgomery R, Logothetis C, et al., Effect of neoadjuvant abiraterone acetate (AA) plus leuprolide acetate (LHRHa) on PSA pathologic response (PCR), and near PCR in localized high-risk prostate cancer (LHRPC): results of a randomized phase II study,
DOI: 10.1200/jco.2012.30.15_suppl.4521, Journal of Clinical Oncology, 30, no. 15_suppl (May 2012) 4521-4521.

12. Fizazi $\mathrm{K}$, Tran $\mathrm{N}$, Fein $\mathrm{L}$, et al., Abiraterone plus prednisone in metastatic, castration-sensitive prostate cancer, N Eng/ / Med, 2017:377:352-60.

13. James ND, DeBona IS, Sears, MR, et al., Abiraterone for prostate cancer not previously treated with hormone therapy, $N$ Eng/ 」 Med, 2017:377:338-51.

14. Kantoff PW, Higano CS, Shore ND, et al., sipuleucelimmunotherapy for castration-resistant prostate cancer, N Eng/ $\mathrm{J}$ Med, 2010;363;411-22.

15. Schellhammer PF, Chodak G, Whitmoree JB, et al., Lower baseline prostate-specific antigen is associated with greater overall survival benefit from sipuleucel-T in the immunotherapy for survival benefit from sipuleucel-T in the immunotherapy for prostate adenocarcin

16. Kantoff PW, Schuetz TJ, Blumenstein BA, et al., Overall survival analysis of a phase II randomized controlled trial of a Poxviralbased PSA targeted immunotherapy in metastatic castration prostate cancer, I Clin Oncol, 2010:28:1099-105.

17. DiPaola RS, Chen YH, Bubly GJ, et al., A national multicenter phase 2 study of prostate-specific antigen (PSA) pox virus vaccine with sequential androgen ablation therapy in patients with PSA progression: ECOG 9802, Eur Urol, 2015;68:365-71.

18. Martin AM, Nirshi TR, Nirshi CJ, et al., Paucity of PD-L1 expression in prostate cancer: innate and adaptive immune resistance, Prostate Cancer Prostatic Dis, 2015;18:325-32.

19. Topalian SL, Hodi FS, Brahmer JR, et al., Safety, activity, and immune correlates of anti-PD-1 antibody in cancer, N Eng/ I Med, 2012;366:2443-54.

20. Bishop JL, Alexander S, Angeles, A et al., PD-L1 is highly expressed in enzalutamide resistant prostate cancer, Oncotarget 2015;6:234-42

21. Graff JN, Alumkal JJ, Drake CG, et al., Early evidence of anti-PD-1 activity in enzautamide-resistant prostate cancer, Oncotarget, 2016;7:52810-7.

22. Modena $A$, Ciccarese $C$, lacovelli $R$, et al., Immune checkpoint inhibitors and prostate cancer: a new frontier?, Oncol Rev, 2016:10:293

23. Kwon $\mathrm{ED}$, Drake $\mathrm{CG}$, Scher HI, et al., I pilimumab versus placebo after radiotherapy in patients with metastatic castration- resistant prostate cancer that had progressed after docetaxel chemotherapy (CA184-043): a multicenter, randomized, doubleblind. Phase 3 trial, Lancet Oncol, 2014;15:700-12.

24. Hodi FS, Chesny J, Pavlick AC et al., Combined nivolumab and ipilimumab versus ipilibumab alone in advanced melanoma: 2-ear overall survival outcomes in a multicentre, randomized, controlled, phase 2 trial, Lancet Oncol, 2016;17:1558-68.

25. Gao J, Ward JF, Pettaway CA, et al., VISTA in an inhibitory immune checkpoint that is increased after ipilimumab therapy in patients with prostate cancer, Nat Med, 2017;23:551-5.

26. Jha GG, Gupta S, Tagawa ST, et al., A phase II randomized, double-blind study of sipuleucel-T followed by IDO pathway inhibitor, indoximod, or placebo in the treatment of patients with metastatic castration-resistant prostate cancer (mCRPC), J Clin Oncol, 2017:35: (suppl; abstr 3066).

27. Crumbaker M, Khoja L, Anthony MJ, AR signaling and the P13K pathway in prostate cancer, Cancers, 2017;9:34

28. Edlind MP, Hsieh AC, P13K-AKT-mTOR signaling in prostate cancer progression and androgen deprivation therapy resistance, Asian Androl, 2014:16:378-86.

29. Taylor BS, Schultz N, Hieronymus $\mathrm{H}$, et al., Integrative genomic profiling of human prostate cancer, Cancer Cell, 2010:18:11-22.

30. Vivanco I, Sawyers Cl, The phosphatidylinositol 3-Kinase AKT pathway in human cancer, Nat Rev Cancer, 2002;2:489-501.

31. Bitting RL, Armstrong AJ, Targeting the P13K mTOR pathway in castration-resistant prostate cancer, Endoc Relat Cancer, 2013;20:R83-99.

32. de Bono JS, DeGiorgi U, Massard C, et al., PTEN loss as a predictive biomarker for Akt inhibitor ipatasertib combined with abiraterone in patients with metastatic castration-resistant prostate cance (mCRPC) Annals of Oncology 2016:27:(suppl6)7180.

33. Robertson D, Van Allen EM, WU YM, et al., Integrative clinical genomics of advanced prostate cancer, Cell, 2015;161:215-28 34. Mateo J, Carreira S, Sandhu S, et al., DNA-repair defects and PTEN-loss as a olaparib in metastatic prostate cancer, N Eng/J Med, 2015;373:1697-708

35. American Cancer Society. Cancer Facts and Figures 2017. Atlanta: American Cancer Society; 2017:p4. Available at: www.cancer.org/ content/dam/cancer-org/research/cancer-facts-and-statistics/ annual-cancer-facts-and-figures/2017/cancer-facts-andfigures-2017.pdf (accessed October 19, 2017) 